\title{
BMJ Open Effectiveness of behavioural change techniques in physiotherapy interventions to promote physical activity adherence in patients with hip and knee osteoarthritis: a systematic review protocol
}

\author{
Matthew Willett, ${ }^{1,2}$ Joan Duda, ${ }^{2,3}$ Charlotte Gautrey, ${ }^{4}$ Sally Fenton, ${ }^{2}$ \\ Carolyn Greig, ${ }^{2,3}$ Alison Rushton ${ }^{1,2}$
}

To cite: Willett M, Duda J, Gautrey C, et al. Effectiveness of behavioural change techniques in physiotherapy interventions to promote physical activity adherence in patients with hip and knee osteoarthritis: a systematic review protocol. BMJ Open 2017;7:e015833. doi:10.1136/ bmjopen-2017-015833

- Prepublication history for this paper is available online. To view these files please visit the journal online (http://dx.doi org/10.1136/bmjopen-2017015833)

Received 3 January 2017 Revised 6 April 2017 Accepted 10 May 2017

CrossMark

For numbered affiliations see end of article.

Correspondence to

Matthew Willett; m.j.willett.1@ bham.ac.uk

\section{ABSTRACT}

Introduction Osteoarthritis $(\mathrm{OA})$ is a common degenerative articular disease, the highest cause of individual level disability and a significant socioeconomic burden to healthcare services. Patient education and physical activity (PA) prescription are recommended components of interventions in several healthcare guidelines and are commonly provided by physiotherapists. However, these interventions lack long-term clinical effectiveness. Patient adherence to PA prescription requires patients to modify their PA behaviour and appears critical in maintaining symptomatic improvements. This systematic review aims to evaluate the effectiveness of behavioural change techniques (BCTs) used in physiotherapy interventions to improve PA adherence.

Methods and analysis Medline, Cochrane and PEDro registers of Controlled Trials, EMBASE, CINAHL and Psyclnfo databases, and key grey literature sources will be rigorously searched for randomised controlled trials that compared a physiotherapy intervention incorporating BCTs with other therapies, placebo interventions, usual care or no-treatment. Two independent researchers will conduct literature searches, assess trial eligibility, extract data, conduct risk of bias assessment (using Cochrane risk of bias tool), classify BCTs and evaluate the quality of the body of literature following Grading of Recommendations, Assessment, Development and Evaluation (GRADE) guidelines. Narrative synthesis of key outcomes will be presented and meta-analysis will be performed if included trials are clinically homogenous, based on their intervention and comparator groups and outcome measures. This review will be reported in line with the Preferred Reporting Items for Systematic review and MetaAnalysis guidelines.

Ethics and dissemination Research ethics approval is not required. This review will help inform clinicians and researchers on the most effective behavioural change techniques used in physiotherapy interventions to enhance adherence to PA prescription for patients with lower limb OA. The findings will be disseminated through
Strengths and limitations of this study

- This systematic review will be the first to rigorously search for, and evaluate the effectiveness of, behavioural change techniques (BCTs), using the behavioural change taxonomy $\mathrm{V} 1$, in physiotherapy randomised controlled trials (RCTs) to promote physical activity $(\mathrm{PA})$ adherence in patients with lower limb osteoarthritis $(\mathrm{OA})$.

- This research will offer physiotherapists and other clinician's evidence-based guidance in selecting BCTs to enhance adherence to PA prescription in patients with lower limb $0 \mathrm{~A}$.

- Several heterogeneous interventions and comparison groups, variabilities in $\mathrm{OA}$ severity, and a limited number of trials are anticipated based on a scoping search. This may preclude metaanalysis, affecting the overall level of evidence for RCT groupings and therefore not enabling firm conclusions on BCT effectiveness to be established.

publication in a peer-reviewed journal and conference presentations.

Trial registration number PROSPERO CRD42016039932.

\section{BACKGROUND}

Osteoarthritis $(\mathrm{OA})$ is a common degenerative disease that causes patients significant pain and reductions in function, social engagement and quality of life. ${ }^{12} \mathrm{OA}$ results in considerable societal healthcare costs and resource utilisation. In $\mathrm{UK}, \mathrm{OA}$ is the most common cause of individual disability, where it is estimated to affect approximately 8.5 million people. ${ }^{3}$ Annually, OA symptoms are estimated to be responsible for approximately 2 million general practitioner visits in the UK, with an expenditure totalling $1 \%$ 
of the country's gross national product. ${ }^{3}$ OA primarily affects the hip and knee synovial joints, with an overall point prevalence of $11 \%$ and $24 \%$, respectively ${ }^{4}$.

Healthcare interventions that incorporate education and physical activity (PA)/exercise prescription are recommended for the non-pharmacological management of $\mathrm{OA}$ in several international guidelines. ${ }^{1}{ }^{3}{ }^{2} 6$ Physiotherapists are commonly the primary healthcare practitioner to whom patients with lower limb OA are referred and are well placed to deliver these interventions. ${ }^{78}$ Although education ${ }^{9}$ and PA interventions ${ }^{10}$ are effective at reducing short-term OA symptoms and clinical outcomes, they lack long-term effectiveness. ${ }^{11}$ With estimates that $50 \%-70 \%$ of patients do not comply with physiotherapy PA recommendations, ${ }^{12}{ }^{13}$ adherence has been identified as a critical reason for this lack of longterm effectiveness. ${ }^{7}$ As OA is a life-long condition, ${ }^{14}$ with point prevalence and incidence increasing with age, ${ }^{4}$ long-term adherence to PA recommendations is critical to maintain the short-term improvements seen in a patient's pain, ${ }^{15}$ function ${ }^{16}$ and disability. ${ }^{17}$

Due to healthcare costs and time constraints, physiotherapy appointments are often limited in number and focus on short-term outcomes only. ${ }^{18}$ As there is usually a gradual decrease in clinical contact time between patient and therapist, long-term PA will most likely continue without supervision in the home/community. ${ }^{19}$ As the positive effects of PA reduce if discontinued, and patient adherence diminishes when physiotherapist supervision ceases, ${ }^{20}$ long-term adherence to recommendations is important and requires patients to change and sustain this change in PA behaviour. ${ }^{20}$

Despite the importance of monitoring PA adherence, at present there is limited evidence to suggest the most appropriate outcomes to measure the maintenance of $\mathrm{PA}$ in patients with lower limb OA. ${ }^{21} \mathrm{~A}$ recent systematic review concluded that no recommendations could be made for any PA adherence outcomes in patients with chronic musculoskeletal pain ${ }^{22}$ due to methodological issues with diagnostic accuracy trial design. Furthermore, none of the seven outcomes identified in the review were validated on patients with OA. A further systematic review identified PA adherence measures used in self-management interventions for patients with musculoskeletal pain. ${ }^{23}$ Six of the 47 trials in the review included participants with lower limb OA, with three of these incorporating exercise diaries and three a multi-item measure to measure PA adherence. ${ }^{23}$

Behavioural change interventions incorporate synchronised techniques that target specific patient health behaviours. ${ }^{24}$ Behavioural change interventions are usually complex ${ }^{25}$ and commonly reported inconsistently in trials, ${ }^{2627}$ making them difficult to replicate in clinical practice. ${ }^{28}$

Incorporating 'active' behavioural techniques (eg, pacing and self-regulatory skills) ${ }^{29}$ into interventions, which encourage patients to participate in their own symptom management has demonstrated greater effectiveness than 'passive' techniques ${ }^{28}$ (provision of information and advice) at maintaining PA behaviours on patients with OA. ${ }^{7}$

Behavioural change techniques (BCTs) are the active components in behaviour al change interventions. ${ }^{30}$ Michie et al (p. 82) define BCTs as 'an observable, replicable and irreducible component of an intervention designed to alter or redirect causal processes that regulate behaviour' and include techniques such as 'reinforcement', 'self-monitoring' and 'feedback'. The identification of BCTs has allowed for specific techniques to be transparently highlighted within interventions and subsequently demonstrated clinical effectiveness. ${ }^{27}$

Intervention fidelity is the degree to which an intervention's active ingredients are delivered as intended. ${ }^{31}$ Intervention fidelity assessment is especially important in behavioural change interventions as it can help determine whether the treatment effect is due to the interacting BCTs or from a variation in the delivery of the intervention protocol. ${ }^{32} 33$ The Behaviour Change Taxonomy $\mathrm{V}^{27}$ has been developed to help authors identify BCTs and improve consistency of reporting, ${ }^{27}$ allowing trials to comply with CONSORT, ${ }^{34}$ Medical Research Council ${ }^{35}$ and 'Template for Intervention Description and Replication' ${ }^{36}$ guidelines for the transparent reporting of interventions and its use should therefore improve intervention fidelity assessment.

Existing PA systematic reviews examining BCTs have focused on broad patient populations, ${ }^{29}{ }^{37-43}$ diabetes, ${ }^{44}$ cardiovascular disease ${ }^{45}$ and rheumatoid arthritis (RA). ${ }^{46}$ To date, only one systematic review ${ }^{40}$ has examined BCT use in physiotherapy practice treating patients with lower limb OA and chronic low back pain with an associated paper assessing each trial's intervention fidelity. ${ }^{33}$ This scoping review identified 33 BCTs used within physiotherapy self-management interventions with no trial (out of $\mathrm{n}=22$ ) demonstrating 'high' treatment fidelity $(>80 \%$ of components present). ${ }^{33}$ Therefore, individual BCT effectiveness was difficult to measure and meta-analysis was not conducted. Furthermore, the grey literature was not searched, meaning up to $10 \%$ of eligible trials were not included, ${ }^{47}$ the review did not target a specified health behaviour (PA adherence) and focused on group classes only. Physiotherapists most commonly treat patients with lower limb (hip and knee) OA individually $(1: 1),{ }^{48}$ and tailoring an intervention to the patients' particular situation is critical to enhance adherence to PA prescription. ${ }^{49}$ Furthermore, recent systematic reviews suggested that 1:1 treatments may provide greater improvements on pain and function than group classes on patients with knee $\mathrm{OA}^{50}$ and $\mathrm{RA},{ }^{46}$ respectively.

Identifying effective BCTs within interventions for patients with chronic conditions is a research priority for the National Institute of Clinical Excellence with 'social support', 'feedback and monitoring' and 'goals and planning', suggested as integral components of programmes to support PA behavioural change. ${ }^{30}$ Additionally, in systematic reviews of PA behaviours, ${ }^{38} 4344$ the BCTs' 
'instruction on how to perform the behaviour', 'demonstration of the behaviour', 'problem-solving' and 'use of follow-up prompts' have been identified as strategies within effective interventions.

As the largest healthcare provider of exercise prescription to patients with musculoskeletal pain in the National Health Service, ${ }^{8}$ physiotherapists are well placed to deliver interventions that incorporate BCTs. However, physiotherapists need to increase their understanding of patients' PA behaviours and motivations to enhance adherence to their recommendations. ${ }^{51}$ Although physiotherapists are encouraged, ${ }^{52}{ }^{53}$ and attempt, ${ }^{54}$ to use behavioural change interventions in their clinical practice, recent evidence suggests that they lack the knowledge base to do so effectively. ${ }^{54}$ A recent systematic review suggested that while incorporating behavioural change into physiotherapy programmes can enhance patient PA adherence, the most effective BCTs have not been determined. ${ }^{55}$ Furthermore, there is a lack of standardised definitions and understanding of BCTs used in physiotherapy interventions when treating patients with $\mathrm{OA}$.

Therefore, the aim of this systematic review is to identify which BCTs used in individual physiotherapy interventions to improve adherence to PA recommendations are most effective in treating patients with lower limb (knee and hip) OA symptoms.

\section{OBJECTIVES}

- To identify BCTs used in individual outpatient physiotherapy interventions to increase or maintain PA adherence outside of the clinic in patients with hip and knee OA.

- To evaluate the clinical effectiveness (on outcomes of PA, adherence, pain, function, quality of life, selfefficacy and adverse effects) of BCTs used in individual outpatient physiotherapy interventions to increase or maintain PA adherence outside of the clinic in patients with hip and knee OA.

\section{METHODS}

This systematic review will be conducted according to a predefined protocol (CRD42016039932) which complies with recommendations from the Cochrane Collaboration Musculoskeletal group ${ }^{56}$ and Centre of Reviews and Dissemination guidelines, ${ }^{57}$ and will be reported following Preferred Reporting Items for Systematic Reviews and Meta-Analysis (PRISMA) guidelines. ${ }^{58}$

\section{Eligibility criteria}

1. Trial design: Randomised controlled trials (RCTs) including protocols, results and fidelity papers where available.

2. Participants: Adult participants ( $\geq 18$ years) with hip and/or knee OA. Diagnosis can be based on acknowledged symptoms, self-reported joint pain or radiographic evidence due to the inconsistency of criteria used across guidelines for hip and knee OA diagnosis. ${ }^{4}$ RCTs whose participants have OA with other chronic coexisting articular pathologies ${ }^{59}$ that contribute $>25 \%$ of their population will be excluded ${ }^{56}$ (eg, septic arthritis, inflammatory joint disease, gout, articular fracture, hemochromatosis). Trials whose participants have had, ${ }^{4960}$ or are awaiting, ${ }^{89}$ surgery for OA treatment (eg, joint arthroplasty) will be excluded, as PA adherence behaviours in this patient population may be different. ${ }^{61}$

3. Interventions:Anystructured outpatient physiotherapy programme that incorporates a BCT that appears on the V1 Taxonomy ${ }^{27}$ as defined by Michie et al that focuses on maintaining or increasing patient PA adherence when away from the physiotherapy clinic (eg, at home or in community). BCTs can include, but are not exclusively: 'prompt self-monitoring of behaviour', 'goal setting', 'social support ${ }^{62}$ and 'reinforcement'. ${ }^{27}$ The intervention must be delivered individually by a physiotherapist (with the profession stated clearly) although follow-up or 'booster' sessions may take different forms (eg, telephone calls). Other members of a multidisciplinary team may be involved in any aspect of the patient's management provided that the physiotherapist is the primary healthcare professional involved and their role can be established by the researcher. Trials that incorporate carers or peer support will be included as long as the patient is the primary target of the physiotherapy intervention.

4. Comparators: Other therapies, placebo interventions, 'no treatment', 'usual care' will be included. RCTs that examined two physiotherapy interventions incorporating BCTs will be included provided that there are different BCTs in each intervention arm, therefore allowing their effectiveness to be determined. RCTs that include cointerventions will be included if the comparison group receives the same cointervention, thereby enabling the effectiveness of the BCTs to be evaluated.

5. Outcomes: It is preferable for trials to have measured PA adherence. Therefore, the main outcomes of interest include PA (eg, pedometers, self-report

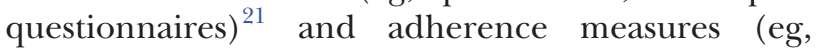
exercise diaries) ${ }^{23}$ although other clinical outcomes of effectiveness (pain, function, quality of life, selfefficacy and adverse effects) will be considered provided they are collected with validated measures. ${ }^{49}$ A note will be made during data extraction whether the trial measured PA adherence specifically and this will interpreted in the discussion section. Only trials that measured PA adherence will be considered for meta-analysis.

6. Language: Trials that are not written in English will be excluded (at full text stage).

\section{Search methods for trial identification}

Medline (OVID) from 1946, the Cochrane Register of Controlled Trials (CENTRAL) from 1940, EMBASE from 
1946, the Physiotherapy Evidence Data base (PEDro) from 1999, Cumulative Index to Nursing and Allied Health Literature (CINAHL) from 1937 and PsycInfo (OVID) from 1806, will be searched. ${ }^{56}$ The clinicaltrial. gov trial register and WHO's trial portal will be searched for relevant trials. ${ }^{56}$ Grey literature will be searched on the 'ZETOC' and 'Conference Proceedings Citation
Index' websites. Reference lists of all included trials and relevant review articles and a citation search using 'web of science' will be conducted. The search strategy for Medline (table 1) has been developed in consultation with a subject-specific librarian and will be adapted for use in other databases. Search terms are informed from recent systematic reviews investigating OA, ${ }^{506364}$ physiotherapy,

Table 1 Search strategy to be used for the MEDLINE electronic database

\begin{tabular}{|c|c|}
\hline Database & Search terms \\
\hline $\begin{array}{l}\text { MEDLINE (OVID) } \\
\text { 1946-present }\end{array}$ & 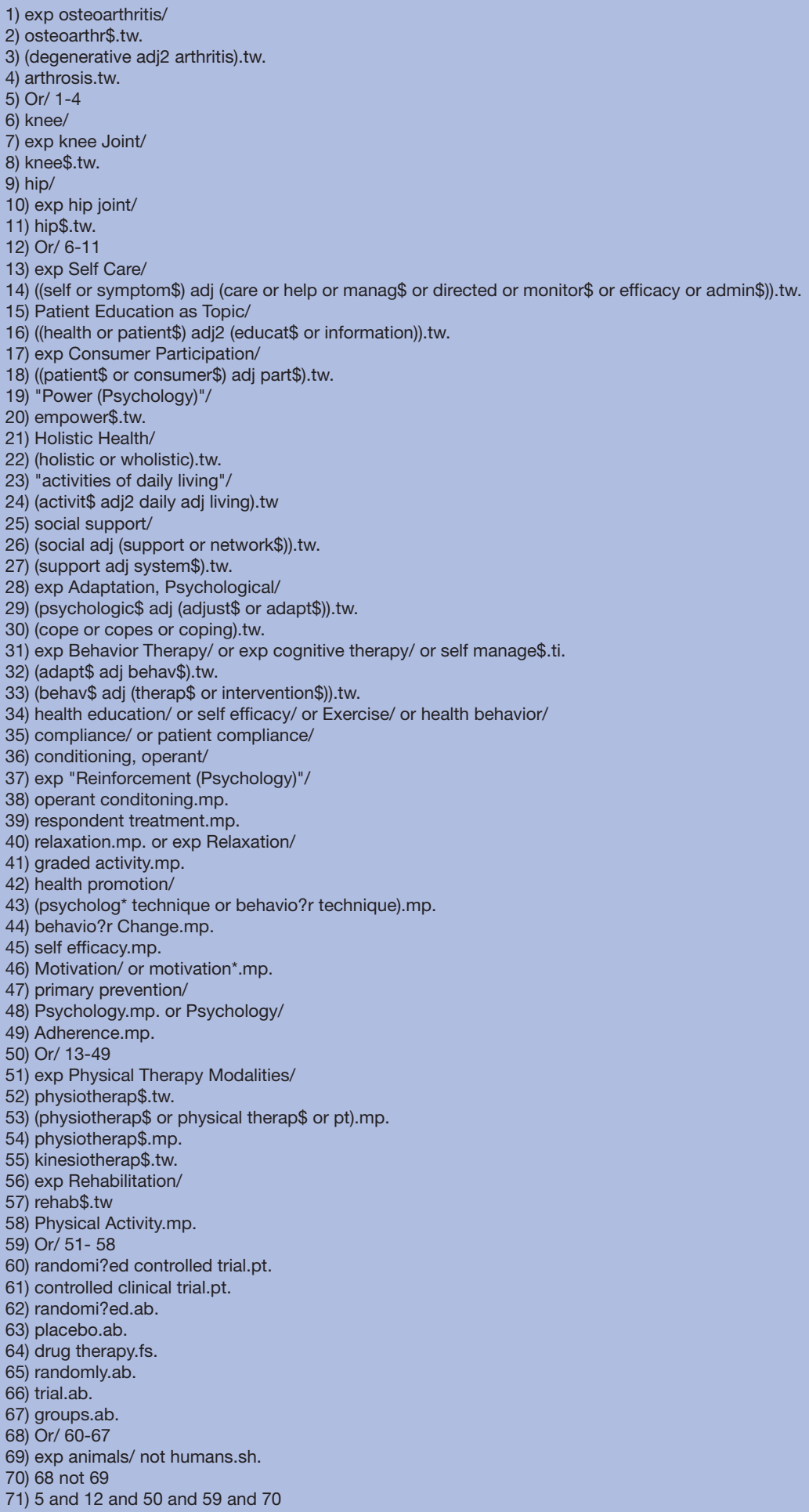 \\
\hline
\end{tabular}


behavioural and education interventions ${ }^{9} 5055$ 63-65 and the scoping search to identify keywords in relevant trials. ${ }^{766}$ RCT filters, as recommended by the Cochrane Collaboration, will be used to prioritise sensitivity over specificity. ${ }^{4767-69}$

\section{Study selection and data management}

Two independent researchers (MJW, ChG) will conduct the initial searches, review abstract and titles, read the full text of included trials or those where uncertainty remains, review relevant reference lists and conduct the citation search. In cases where the two researchers cannot agree on eligibility, a third researcher (AR, subject and methodological expertise) will mediate. Initial search results will be uploaded to Refworks prior to the review of titles and abstracts. Included trials will be managed through EndNote. A PRISMA flow chart will be used to provide transparency of the number of trials included or excluded at each stage.

\section{Data collection process and analyses}

Two independent researchers (MJW, SF) will use a standardised data extraction form developed from the Cochrane Back and Neck group template to record information on participants, trial setting, eligibility criteria, risk of bias assessment, methodology design, intervention, outcome measures (with special attention on PA adherence measures), assessment time points, PA adherence BCTs within the intervention, the deliverer of BCTs and any training they undertook and the main trial results. ${ }^{70}$

Data extraction will include detail on trial treatment fidelity. Although several checklists exist to assess intervention fidelity, ${ }^{71}$ The National Institutes of Health Behaviour Change Consortium's (NIHBCC) checklist is unique in its focus on behavioural change trials and has demonstrated validity and reliability. ${ }^{32}$ The NIHBCC checklist has 40 components and was developed in $2011^{73}$ from the initial version which had 25 components. ${ }^{32}$ The NIHBCC's checklist comprises five domains: 'Treatment design', 'Training Providers', 'Delivery of Treatment', 'Receipt of Treatment' and 'Enactment of Treatment skills'. Although Toomey et $a l^{33}$ did not find any association between trial date and fidelity, the consideration and assessment of fidelity is a relatively contemporary concept and the scoping search revealed several trials conducted prior, or at a similar time, to the NIHBCC checklists creation. While it is not the primary research question in this systematic review, trials treatment fidelity needs to be acknowledged when determining intervention effectiveness. Therefore, in order not to overtly penalise trials but to aid interpretation, each domain on the NIHBCC checklist will be judged as 'present' or 'absent' by two independent researchers (MJW, SF) but no score will be given to individual items. These details will be included as part of the narrative synthesis and interpreted in the discussion when drawing conclusions regarding BCT effectiveness.
The data extraction form will be piloted on the full texts of several included RCTs to ensure reliability and will be altered as necessary to optimise data collection. Any disagreement between researchers will be resolved through discussion. If agreement cannot be reached, a third reviewer (AR) will mediate. Where there are multiple reports of the same trial, data will be extracted using separate forms and collated on a single form subsequently. ${ }^{70}$ Trial authors will be contacted by email if information is missing or unclear.

Two independent researchers (MJW, SF) will code interventions using the 'Behaviour Change Taxonomy'. ${ }^{27}$ BCTs and their associated hierarchy will be included as a component of the data extraction. As per training instructions, the associated text and page number will be recorded and the BCT will be given a 'score' of + (present in all probability) or ++ (present beyond all reasonable doubt) ${ }^{74}$ to facilitate further discussion. Only BCTs that are directed at PA adherence behaviour will be coded. ${ }^{74}$ Trials that have available protocols and fidelity papers will also be coded. ${ }^{40}$ The researchers will undergo online training on the use of the taxonomy and the coding process will be piloted a priori. To ensure that a 'postlearning effect ${ }^{, 75}$ is minimised, a period of integration will be observed after the training, and coders will meet regularly to minimise discrepancies in taxonomy understanding and enhance agreement. Taxonomy use will be piloted a priori, and coder agreement will be calculated using Cohen's Kappa statistic. Any disagreement between the researchers will be resolved by researcher consensus. In the event that consensus cannot be reached, a third researcher (JD, expertise in subject area of behavioural change) will mediate the decision (objective 1).

\section{Risk of bias assessment}

Two independent researchers (MJW, SF) will use the Cochrane Risk of Bias tool to assess the internal validity of included trials. ${ }^{76}$ The tool was developed by a research working group and is recommended for use in systematic reviews. It uses domain-based evaluation rather than a check list or scoring system to assess internal validity ${ }^{76}$ and allows review authors space to justify their conclusions. ${ }^{77}$ The tool addresses six domains: 'sequence generation', 'allocation concealment', 'blinding', 'incomplete outcome data', 'selective outcome reporting' and 'other' sources of bias. A judgement of 'high', 'unclear' or 'low' risk of bias will be made for each domain. A judgement of 'Unclear' will be allocated to a domain where insufficient information is provided. Trials will be screened for selective outcome reporting by comparing outcomes used in the finalised articles with registered protocols. If no trial protocol exists, outcomes from the trials published methods and results sections will be compared and a judgement of 'unclear' will be allocated ${ }^{58}$ When assessing trials risk of bias, researchers will pay special attention to the 'blinding' domain. Blinding of the treating physiotherapists and trial participants is often problematic; however, assessor blinding is achievable and important. ${ }^{76}$ 
Therefore, trials will be judged to have overall 'low risk' for the blinding domain if the assessor is adequately blinded. ${ }^{76}$ The risk of bias assessment across trials will be displayed graphically using REVMAN 5.3.

\section{Data presentation}

A table will be presented that details the BCTs used in each trial. The total number of BCTs (individually and hierarchal groups) used across trials, their frequency per trial and how accurately they were reported will be detailed (objective 1). ${ }^{40} \mathrm{~A}$ 'risk of bias' table showing internal validity decisions within and across trials will also be presented. A 'characteristics of included trials' table with PICOS data with explicit detail noting:

- Intervention: fidelity assessment, whether other intervention providers were involved in BCT delivery and any training in physiotherapist BCT delivery.

- Outcomes: Trials that used a PA adherence measure.

\section{Data synthesis}

Narrative synthesis will be reported following stages as recommended by Cochrane Qualitative Research Group. $^{78}$

\section{Developing a preliminary synthesis}

Trials $(\geq 2)$ will be grouped together if they are clinically homogenous as determined by two researchers (MJW, $\mathrm{SF})$, based on:

- Interventions: specific BCTs with or without cointerventions

- Comparator groups

- Outcome measure domains ${ }^{79}$

The results of groupings will be presented in tables (objective 1).

\section{Assessing the robustness of the synthesis}

Each table and statement will include information detailing overall quality of evidence for each grouping. The quality of the body of evidence for each outcome will be evaluated using the GRADE approach (Grading of Recommendations, Assessment, Development and Evaluation) ${ }^{80}$ GRADE determines overall quality of evidence based on risk of bias, inconsistency, indirectness, imprecision of results and publication bias. ${ }^{80}$ The quality of evidence will be adjudicated as 'high', 'moderate', 'low' or 'very low' based on the guidance from the GRADE working group by two researchers (MJW, SF), ${ }^{80}$ with a third researcher (AR) asked to mediate if consensus cannot be reached.

\section{Exploring relationships within and between trials}

Further textual descriptions will accompany the tables to highlight key points on trial population, BCTs identified and accuracy of reporting, intervention fidelity assessment, the BCT intervention provider and their training or outcome measures that could explain the differences in results as outlined in the tables.

\section{BCT effectiveness}

Individual BCT effectiveness will be determined narratively and supported quantitatively by the use of its 'percentage effectiveness ratio'. ${ }^{81}$ Trial interventions will be categorised as 'effective' or 'ineffective' with effective trials demonstrating a significantly greater effect on PA or adherence outcome measures when displayed in a forest plot. The ratio will be calculated by dividing the number of times that the BCT was part of an 'effective' intervention by the number of times the BCT was used in all trials (objective 2) ${ }^{81}$

\section{Meta-analysis}

Based on the scoping search, it is anticipated that eligible trials will demonstrate high intervention, comparator and outcome variability (clinical heterogeneity) ${ }^{79}$ Therefore, when meta-analysis is indicated, a random effects model will be used to calculate effect sizes based on the groupings outlined in the narrative synthesis (objective 2). The risk ratio with $95 \%$ CIs and mean differences (MD) with 95\% CIs will be used to measure the treatment effect of dichotomous and continuous outcomes, respectively. The standardised mean difference will be used to measure continuous outcomes where several measures are used within one outcome domain. ${ }^{79}$ Where mean data are not available and trial authors do not respond to an email request for raw data (a maximum of three follow-up emails), the median will be used as an estimate of the mean. $^{82}$

\section{Sensitivity analysis}

A sensitivity analysis will be conducted to determine whether excluding high risk of bias RCTs has influenced the findings of the meta-analysis to enable further discussion (objective 2).

\section{Meta-bias}

Publication bias will be assessed by use of funnel plots where meta-analysis includes $\geq 10$ trials. ${ }^{83}$ Meta-analyses will be tested for 'small-study effects' by comparing fixed and random effects sizes where the random-effects model will show greater intervention effect sizes for trials with smaller sample sizes. ${ }^{83}$

\section{DISCUSSION}

Patients with OA currently display the highest level of individual level disability in the UK. Interventions incorporating BCTS have the potential to increase long-term patient adherence to PA recommendations, increasing patient function and quality of life, and physiotherapists are well placed to deliver them. At present, there is a lack of understanding of specific BCTs used in physiotherapy interventions when treating patients with $\mathrm{OA}$. This review will help identify BCTs currently being used in physiotherapy clinical practice and recommend those which are the most effective at reducing lower limb OA symptoms and encouraging long-term patient PA adherence. Clinicians will be able to apply the evidence from this 
systematic review on patients with OA by incorporating the most appropriate BCTs into their interventions to maximise their adherence to PA. This systematic review will also inform the planning and implementation of a trial to determine the feasibility of an active behavioural physiotherapy intervention on patients with lower limb OA.

\section{LIMITATIONS}

Several heterogeneous interventions, comparison groups, variability of $\mathrm{OA}$ severity and time periods and a limited number of trials (15-20) are anticipated based on the scoping search. This may preclude meta-analysis, affecting the overall level of evidence for RCT groupings. Furthermore, the fidelity assessment and BCTs within interventions may be poorly reported, making it difficult to determine the effectiveness of individual BCTs with consideration of the degree to which the intervention was delivered as intended.

\section{ETHICS AND DISSEMINATION}

No research ethics approval is required for this systematic review as no confidential patient data will be used. It is intended that the results of this systematic review will be disseminated through publication in a peer reviewed journal and conference presentations.

\section{Author affiliations}

${ }^{1}$ Centre of Precision Rehabilitation for Spinal Pain (CPR Spine), School of Sport, Exercise and Rehabilitation Sciences, University of Birmingham, Birmingham, UK ${ }^{2}$ School of Sport, Exercise and Rehabilitation Sciences, University of Birmingham, Birmingham, UK

${ }^{3}$ MRC-Arthritis Research UK Centre for Musculoskeletal Ageing Research, University of Birmingham, Birmingham, UK

${ }^{4}$ School of Life and Medical Sciences, University of Hertfordshire, Hatfield, UK

Contributors All authors conceived the focus of the systematic review. MW is a $\mathrm{PhD}$ student and AR (lead supervisor), JD and CaG are supervisors. MW drafted the initial version of the protocol manuscript. AR, CaG and JD provided critical guidance on the direction, methodological decisions and proposed analyses. MW developed the search strategy and data extraction form that was piloted by MW and ChG/SF, respectively. All authors reviewed and commented on each draft of the systematic review protocol. MW is the guarantor of the review.

Funding This research received no specific grant from any funding agency in the public, commercial or not-for-profit sectors.

Competing interests None declared

Provenance and peer review Not commissioned; externally peer reviewed.

Data sharing statement This systematic review protocol was registered with the International Prospective Register of Systematic Reviews (PROSPERO) on 15/8/2016 Registration number: CRD42016039932. This protocol has been reported using the Preferred Reporting Items for Systematic Reviews and MetaAnalyses Protocols (PRISMA-P) guidelines. The final version of this systematic review and its associated data will be published on completion.

Open Access This is an Open Access article distributed in accordance with the Creative Commons Attribution Non Commercial (CC BY-NC 4.0) license, which permits others to distribute, remix, adapt, build upon this work non-commercially, and license their derivative works on different terms, provided the original work is properly cited and the use is non-commercial. See: http://creativecommons.org/ licenses/by-nc/4.0/

(C) Article author(s) (or their employer(s) unless otherwise stated in the text of the article) 2017. All rights reserved. No commercial use is permitted unless otherwise expressly granted.

\section{REFERENCES}

1. Fernandes L, Hagen KB, Bijlsma JW, et al. EULAR recommendations for the non-pharmacological core management of hip and knee osteoarthritis. Ann Rheum Dis 2013;72:1125-35.

2. Helminen EE, Sinikallio SH, Valjakka AL, et al. Effectiveness of a cognitive-behavioural group intervention for knee osteoarthritis pain: a randomized controlled trial. Clin Rehabil 2015;29:868-81.

3. Osteoarthritis N. National Istitute for for Health and Care Excellence 2014. Care and Management in adults. clinical guideline CG177 methods, evidence and recommendations, 2014.

4. Pereira D, Peleteiro B, Araújo J, et al. The effect of osteoarthritis definition on prevalence and incidence estimates: a systematic review. Osteoarthritis Cartilage 2011;19:1270-85.

5. Hochberg MC, Altman RD, April KT, et al. American College of Rheumatology 2012 recommendations for the use of nonpharmacologic and pharmacologic therapies in osteoarthritis of the hand, hip, and knee. Arthritis Care Res 2012;64:465-74.

6. McAlindon TE, Bannuru RR, Sullivan MC, et al. OARSI guidelines for the non-surgical management of knee osteoarthritis. Osteoarthritis Cartilage 2014;22:363-88.

7. Pisters MF, Veenhof $\mathrm{C}$, de Bakker $\mathrm{DH}$, et al. Behavioural graded activity results in better exercise adherence and more physical activity than usual care in people with osteoarthritis: a clusterrandomised trial. J Physiother 2010;56:41-7.

8. Foster NE, Healey EL, Holden MA, et al. A multicentre, pragmatic, parallel group, randomised controlled trial to compare the clinical and cost-effectiveness of three physiotherapy-led exercise interventions for knee osteoarthritis in older adults: the BEEP trial protocol (ISRCTN: 93634563). BMC Musculoskelet Disord 2014;15:254.

9. Kroon FPB, Van der Burg L, Buchbinder R, et al. The Cochrane Library. Self-management education programmes for osteoarthritis, 2014.

10. Pisters MF, Veenhof C, Schellevis FG, et al. Long-term effectiveness of exercise therapy in patients with osteoarthritis of the hip or knee: a randomized controlled trial comparing two different physical therapy interventions. Osteoarthritis Cartilage 2010;18:1019-26.

11. Bennell KL, Dobson F, Hinman RS. Exercise in osteoarthritis: moving from prescription to adherence. Best Pract Res Clin Rheumatol 2014;28:93-117.

12. Beinart NA, Goodchild CE, Weinman JA, et al. Individual and intervention-related factors associated with adherence to home exercise in chronic low back pain: a systematic review. Spine $J$ 2013;13:1940-50.

13. Bassett SF. The assessment of patient adherence to physiotherapy rehabilitation. New Zealand Journal of Physiotherapy 2003;31:60-6.

14. Thorstensson CA, Garellick G, Rystedt $\mathrm{H}$, et al. Better Management of Patients with Osteoarthritis: development and Nationwide Implementation of an Evidence-Based Supported Osteoarthritis SelfManagement Programme. Musculoskeletal Care 2015;13:67-75.

15. Thomas KS, Muir KR, Doherty M, et al. Home based exercise programme for knee pain and knee osteoarthritis: randomised controlled trial. BMJ 2002;325:752-6.

16. Dunlop DD, Semanik P, Song J, et al. Risk factors for functional decline in older adults with arthritis. Arthritis Rheum 2005;52:1274-82.

17. Marks R, Allegrante JP. Chronic osteoarthritis and adherence to exercise: a review of the literature. J Aging Phys Act 2005;13:434-60.

18. Holden MA, Nicholls EE, Hay EM, et al. Physical therapists' use of therapeutic exercise for patients with clinical knee osteoarthritis in the United kingdom: in line with current recommendations? Phys Ther 2008;88:1109-21.

19. van Gool CH, Penninx BW, Kempen Gl, et al. Effects of exercise adherence on physical function among overweight older adults with knee osteoarthritis. Arthritis Rheum 2005;53:24-32.

20. Hinman RS, Delany CM, Campbell PK, et al. About Working Together to Promote Exercise Patients With Knee Osteoarthritis: Qualitative Study Physical Therapists, Telephone Coaches, and Adherence. Physical Therapy 2015;96:479-93.

21. Terwee CB, Bouwmeester W, van Elsland SL, et al. Instruments to assess physical activity in patients with osteoarthritis of the hip or knee: a systematic review of measurement properties. Osteoarthritis Cartilage 2011;19:620-33.

22. McLean S, Holden MA, Potia T, et al. Quality and acceptability of measures of exercise adherence in musculoskeletal settings: a systematic review. Rheumatology 2017;56:kew422.

23. Hall AM, Kamper SJ, Hernon M, et al. Measurement tools for adherence to non-pharmacologic self-management treatment for chronic musculoskeletal conditions: a systematic review. Arch Phys Med Rehabil 2015;96:552-62.

24. Michie S, van Stralen MM, West R. The behaviour change wheel: a new method for characterising and designing behaviour change interventions. Implement Sci 2011;6:42. 
25. Craig P, Dieppe P, Macintyre S, et al. Developing and evaluating complex interventions: the New Medical Research Council guidance. BMJ 2008;337:a1655.

26. Michie S, Johnston M, Francis J, et al. From Theory to intervention: mapping theoretically derived Behavioural Determinants to Behaviour Change Techniques. Appl Psychol 2008;57:660-80.

27. Michie S, Richardson M, Johnston M, et al. The behavior change technique taxonomy ( $v 1)$ of 93 hierarchically clustered techniques: building an international consensus for the reporting of behavior change interventions. Ann Behav Med 2013;46:81-95.

28. Albarracín D, Gillette JC, Earl AN, et al. A test of Major assumptions about behavior change: a comprehensive look at the effects of passive and active HIV-prevention interventions since the beginning of the epidemic. Psychol Bull 2005;131:856-97.

29. Michie $S$, Abraham $C$, Whittington $C$, et al. Effective techniques in healthy eating and physical activity interventions: a meta-regression. Health Psychol 2009;28:690-701.

30. NICE. PH49:Behaviour change: individual approaches. National Istitute for for Health and Care Excellence, 2014.

31. Toomey E, Matthews J, Guerin S, et al. Development of a Feasible Implementation Fidelity Protocol Within a Complex Physical TherapyLed Self-Management Intervention. Phys Ther 2016;96:1287-98.

32. Borrelli B, Sepinwall D, Ernst D, et al. A new tool to assess treatment fidelity and evaluation of treatment fidelity across 10 years of health behavior research. J Consult Clin Psychol 2005;73:852-60.

33. Toomey E, Currie-Murphy L, Matthews J, et al. Implementation fidelity of physiotherapist-delivered group education and exercise interventions to promote self-management in people with osteoarthritis and chronic low back pain: a rapid review part II. Man Ther 2015;20:287-94.

34. Boutron I, Moher D, Altman DG, et al. Extending the CONSORT statement to randomized trials of nonpharmacologic treatment: explanation and elaboration. Ann Intern Med 2008;148:295-309.

35. Craig P, Dieppe P, Macintyre S, et al. Developing and evaluating complex interventions: new guidance. Medical Research Council 2008.

36. Hoffmann TC, Glasziou PP, Boutron I, et al. Better reporting of interventions: template for intervention description and replication (TIDieR) checklist and guide. BMJ 2014;348:g1687.

37. Bird EL, Baker G, Mutrie N, et al. Behavior change techniques used to promote walking and cycling: a systematic review. Health Psychol 2013;32:829-38.

38. French DP, Olander EK, Chisholm A, et al. Which behaviour change techniques are most effective at increasing older adults' self-efficacy and physical activity behaviour? A systematic review. Ann Behav Med 2014;48:225-34

39. Plow MA, Moore S, Husni ME, et al. A systematic review of behavioural techniques used in nutrition and weight loss interventions among adults with mobility-impairing neurological and musculoskeletal conditions. Obes Rev 2014;15:945-56.

40. Keogh A, Tully MA, Matthews J, et al. A review of behaviour change theories and techniques used in group based self-management programmes for chronic low back pain and arthritis. Man Ther 2015;20:727-35.

41. O'Brien N, McDonald S, Araújo-Soares V, et al. The features of interventions associated with long-term effectiveness of physical activity interventions in adults aged 55-70 years: a systematic review and meta-analysis. Health Psychol Rev 2015;9:417-33.

42. Olander EK, Fletcher $\mathrm{H}$, Williams $\mathrm{S}$, et al. What are the most effective techniques in changing obese individuals' physical activity selfefficacy and behaviour: a systematic review and meta-analysis. Int $J$ Behav Nutr Phys Act 2013;10:29.

43. Dombrowski SU, Sniehotta FF, Avenell A, et al. Identifying active ingredients in complex behavioural interventions for obese adults with obesity-related co-morbidities or additional risk factors for comorbidities: a systematic review. Health Psychol Rev 2012;6:7-32.

44. Cradock KA, ÓLaighin G, Finucane FM, et al. Behaviour change techniques targeting both diet and physical activity in type 2 diabetes: A systematic review and meta-analysis. Int J Behav Nutr Phys Act 2017:14:18.

45. Heron N, Kee F, Donnelly M, et al. Behaviour change techniques in home-based cardiac rehabilitation: a systematic review. Br J Gen Pract 2016:66:e747-e757.

46. Cramp F, Berry J, Gardiner M, et al. Health behaviour change interventions for the promotion of physical activity in rheumatoid arthritis: a systematic review. Musculoskeletal Care 2013;11:238-47.

47. Lefebvre C, Manheimer E, Glanville J. The Cochrane Collaboration, 2011. Chapter 6: Searching for studies. In: Higgins JPT, Green $\mathrm{S}$, Cochrane Handbook for Systematic Reviews of Interventions Version 5.1.0, 2011. www.handbook.cochrane.org (updated Mar 2011).
48. Tiffreau V, Mulleman D, Coudeyre E, et al. The value of individual or collective group exercise programs for knee or hip osteoarthritis. elaboration of French clinical practice guidelines. Annales de R?adaptation et de M?decine Physique 2007;50:741-6.

49. Jordan JL, Holden MA, Mason EEJ, et al. Interventions to improve adherence to exercise for chronic musculoskeletal pain in adults. The Cochrane Library 2010.

50. Fransen M, McConnell S, Harmer AR, et al. Exercise for osteoarthritis of the knee. Cochrane Database of Systematic Reviews 20152015.

51. Chan DK, Lonsdale C, Ho PY, et al. Patient motivation and adherence to postsurgery rehabilitation exercise recommendations: the influence of physiotherapists' autonomy-supportive behaviors. Arch Phys Med Rehabil 2009;90:1977-82.

52. DOH. The NHS knowledge skills framework and the development review process. NHS. 2004, 2004.

53. HCPC. Standards of proficiency for physiotherapists. NHS. , 2013:2013, 1-18

54. Alexanders J, Anderson A, Henderson S. Musculoskeletal physiotherapists' use of psychological interventions: a systematic review of therapists' perceptions and practice. Physiotherapy 2015;101:95-102.

55. McGrane N, Galvin R, Cusack T, et al. Addition of motivational interventions to exercise and traditional physiotherapy: a review and meta-analysis. Physiotherapy 2015;101:1-12

56. Ghogomu EA, Maxwell LJ, Buchbinder R, et al. Updated method guidelines for cochrane musculoskeletal group systematic reviews and metaanalyses. J Rheumatol 2014;41:194-205.

57. CRD. Centre for Reviews and Dissemination (CRD). Systematic reviews: crd's guidance for undertaking reviews in healthcare. 3rd ed. York: CRD University of York, York Publishing Services Ltd, 2009.

58. Shamseer L, Moher D, Clarke M, et al. Preferred reporting items for systematic review and meta-analysis protocols (PRISMA-P) 2015: elaboration and explanation. BMJ 2015;349:g7647.

59. McAlindon TE, Driban JB, Henrotin Y, et al. OARSI clinical trials recommendations: design, conduct, and reporting of clinical trials for knee osteoarthritis. Osteoarthritis Cartilage 2015;23:747-60.

60. Brand E, Nyland J, Henzman C, et al. Arthritis Self-Efficacy Scale scores in knee osteoarthritis: a systematic review and meta-analysis comparing arthritis self-management education with or without exercise. J Orthop Sports Phys Ther 2013;43:895-910.

61. Dobson F, Bennell KL, French SD, et al. Barriers and Facilitators to Exercise Participation in People with Hip and/or Knee Osteoarthritis: Synthesis of the Literature Using Behavior Change Theory. Am J Phys Med Rehabil 2016;95:372-89.

62. Marley J, Tully MA, Porter-Armstrong A, et al. A systematic review of interventions aimed at increasing physical activity in adults with chronic musculoskeletal pain--protocol. Syst Rev 2014;3:106.

63. Fransen M, McConnell S, Hernandez-Molina G, et al. Exercise for osteoarthritis of the hip. Cochrane Database Syst Rev 2014;2014.

64. French HP, Galvin RP, Abbott JH, et al. The Cochrane Library. Adjunctive therapies in addition to land-based exercise therapy for osteoarthritis of the hip or knee, 2015.

65. Toomey E, Currie-Murphy L, Matthews J, et al. The effectiveness of physiotherapist-delivered group education and exercise interventions to promote self-management for people with osteoarthritis and chronic low back pain: a rapid review part I. Man Ther 2015:20:265-86.

66. Veenhof C, Köke AJ, Dekker J, et al. Effectiveness of behavioral graded activity in patients with osteoarthritis of the hip and/or knee: A randomized clinical trial. Arthritis Rheum 2006;55:925-34.

67. Wong SS, Wilczynski NL, Haynes RB. Developing optimal search strategies for detecting clinically sound treatment studies in EMBASE. J Med Libr Assoc 2006;94:41-7.

68. Wong SS, Wilczynski NL, Haynes RB. Optimal CINAHL search strategies for identifying therapy studies and review articles. J Nurs Scholarsh 2006;38:194-9.

69. Eady AM, Wilczynski NL, Haynes RB. PsycINFO search strategies identified methodologically sound therapy studies and review articles for use by clinicians and researchers. J Clin Epidemiol 2008;61:34-40.

70. Higgins JPT, Deeks JJ. The Cochrane Collaboration, 2011. Chapte 7: Selecting studies and collecting data. In: Higgins JPT, Green S, eds. Cochrane Handbook for Systematic Reviews of Interventions Version 5.1.0, 2011. www.handbook.cochrane.org (updated Mar 2011).

71. Carroll C, Patterson M, Wood S, et al. A conceptual framework for implementation fidelity. Implement Sci 2007;2:40.

72. Gearing RE, El-Bassel N, Ghesquiere A, et al. Major ingredients of fidelity: a review and scientific guide to improving quality of intervention research implementation. Clin Psychol Rev 2011;31:79-88 
73. Borrelli B. The Assessment, Monitoring, and Enhancement of Treatment Fidelity In Public Health Clinical Trials. J Public Health Dent 2011;71:S52-S63.

74. Michie S, Johnston M, Abraham C, et al. BCT Taxonomy: Starter Pack For trainees, 2014

75. Wood CE, Hardeman W, Johnston M, et al. Reporting behaviour change interventions: do the behaviour change technique taxonomy $\mathrm{v} 1$, and training in its use, improve the quality of intervention descriptions? Implement Sci 2016;11:84.

76. Higgins JPT, Altman DG, Sterne JAC. The Cochrane Collaboration, 2011. Chapter 8: Assessing risk of bias in included studies. In: Higgins JPT, Green S, eds. Cochrane Handbook for Systematic Reviews of Interventions Version 5.1.0, 2011. www.handbook. cochrane.org (updated Mar 2011).

77. Liberati A, Altman DG, Tetzlaff J, et al. The PRISMA statement for reporting systematic reviews and meta-analyses of studies that evaluate healthcare interventions: explanation and elaboration. BMJ 2009;339:b2700.

78. Popay J, Roberts H, Sowden A, et al. Guidance on the conduct of narrative synthesis in systematic reviews. A product from the ESRC methods Programme. Version 1: ESRC, 2006:1-91.
79. Deeks JJ, Higgins JPT, Altman DG. The Cochrane Collaboration, 2011. Chapter 9: Analysing data and undertaking meta-analyses. In: Higgins JPT, Green S, Cochrane Handbook for Systematic Reviews of Interventions Version 5.1.0. www.handbook.cochrane.org. 2011. (updated Mar 2011)

80. Schünemann HJ, Oxman AD, Vist GE, et al. Chapter 12: Interpreting results and drawing conclusions. In: Higgins JPT, Green S, Cochrane Handbook for Systematic Reviews of Interventions Version 5.1.0. The Cochrane Collaboration, 2011. www.handbook.cochrane.org (updated mar 2011)

81. Martin J, Chater A, Lorencatto F. Effective behaviour change techniques in the prevention and management of childhood obesity. Int J Obes 2013;37:1287-94.

82. Hozo SP, Djulbegovic B, Hozo I. Estimating the mean and variance from the median, range, and the size of a sample. BMC Med Res Methodol 2005;5:13

83. Sterne JA, Sutton AJ, loannidis JP, et al. Recommendations for examining and interpreting funnel plot asymmetry in meta-analyses of randomised controlled trials. BMJ 2011;343:d4002. 